\title{
Application of metabolomics: Focus on the quantification of organic acids in healthy adults
}

\author{
DIMITRIS TSOUKALAS ${ }^{1,2 *}$, ATHANASIOS ALEGAKIS ${ }^{1 *}$, PERSEFONI FRAGKIADAKI $^{1 *}$, \\ EVANGELOS PAPAKONSTANTINOU ${ }^{2}$, DRAGANA NIKITOVIC ${ }^{3}$, AIKATERINI KARATARAKI ${ }^{2}$, \\ ALEXANDER E. NOSYREV ${ }^{4}$, EMMANOUEL G. PAPADAKIS ${ }^{5}$, DEMETRIOS A. SPANDIDOS ${ }^{6}$, \\ NIKOLAOS DRAKOULIS ${ }^{7}$ and ARISTIDES M. TSATSAKIS ${ }^{1}$
}

\author{
${ }^{1}$ Laboratory of Forensic Sciences and Toxicology, Medical School, University of Crete, Heraklion 71003; \\ ${ }^{2}$ Metabolomic Medicine Clinic, Athens 10674; ${ }^{3}$ Laboratory of Anatomy-Histology-Embryology, School of Medicine, \\ University of Crete, Heraklion 71003, Greece; ${ }^{4}$ I.M. Sechenov First Moscow State Medical University, Moscow 119048, \\ Russia; ${ }^{5}$ Onassis Cardiac Surgery Centre, Athens 17674; ${ }^{6}$ Laboratory of Clinical Virology, School of Medicine, \\ University of Crete, Heraklion 71003; ${ }^{7}$ Research Group of Clinical Pharmacology and Pharmacogenomics, \\ Faculty of Pharmacy, School of Health Sciences, National and Kapodistrian University of Athens, Athens 15772, Greece
}

Received February 22, 2017; Accepted May 2, 2017

DOI: $10.3892 /$ ijmm.2017.2983

\begin{abstract}
Metabolomics, a 'budding' discipline, may accurately reflect a specific phenotype which is sensitive to genetic and epigenetic interactions. This rapidly evolving field in science has been proposed as a tool for the evaluation of the effects of epigenetic factors, such as nutrition, environment, drug and lifestyle on phenotype. Urine, being sterile, is easy to obtain and as it contains metabolized or non-metabolized products, is a favored study material in the field of metabolomics. Urine organic acids (OAs) reflect the activity of main metabolic pathways and have been used to assess health status, nutritional status, vitamin deficiencies and response to xenobiotics. To date, a limited number of studies have been performed which actually define reference OA values in a healthy population and as reference range for epigenetic influences, and not as a reference to congenital metabolic diseases. The aim of the present study was thus the determination of reference values (RVs) for urine OA in a healthy adult population. Targeted metabolomics analysis of 22 OAs in the urine of 122 healthy adults by gas
\end{abstract}

Correspondence to: Dr Nikolaos Drakoulis, Research Group of Clinical Pharmacology and Pharmacogenomics, Faculty of Pharmacy, School of Health Sciences, National and Kapodistrian University of Athens, Panepistimiopolis Zografou, Athens 15772, Greece

E-mail: drakoulis@pharm.uoa.gr

Professor Aristides M. Tsatsakis, Laboratory of Forensic Sciences and Toxicology, Medical School, University of Crete, P.O. Box 1393, Heraklion 71003, Greece

E-mail: toxlab.uoc@gmail.com

*Contributed equally

Key words: organic acid, metabolomic, vitamin deficiencies, xenobiotics, adults chromatography-mass spectrometry, was conducted. Percentile distributions of the OA concentrations in urine, as a base for determining the RVs in the respective population sample, were used. No significant differences were detected between female and male individuals. These findings can facilitate the more sensitive determination of OAs in pathological conditions. Therefore, the findings of this study may contribute or add to the information already available on urine metabolite databases, and may thus promote the use of targeted metabolomics for the evaluation of OAs in a clinical setting and for pathophysiological evaluation. However, further studies with well-defined patients groups exhibiting specific symptoms or diseases are warranted in order to discern between normal and pathological values.

\section{Introduction}

Metabolomics is the youngest of the emerging 'omics' sciences and along with 'sister' genomics, transcriptomics and proteomics, comprises a Systems Biology approach with a potential significant role in personalized medicine $(1,2)$ While genomics relates to the genotype and metabolomics reflects the phenotype, recent studies have demonstrated that the association of genetic polymorphisms and consequent metabolite changes may provide unique individual genetic and phenotype information towards personalized medicine, and may reflect individual responses to lifestyle and epigenetic factors $(3,4)$. Organic acids (OAs) are a particular group of metabolites, which have gained increasing scientific interest. Indeed, these organic compounds are intermediate metabolites of critical metabolic pathways, such as the Krebs cycle, carbohydrate metabolism, ketone body metabolism, fatty acid $\beta$-oxidation, neurotransmitters turnover and protein metabolism. The determination of OAs in urine has been utilized, from the early study by Tanaka and Isselbacher, who documented the first organic aciduria in 1970 (5). This breakthrough finding was revolutionarily utilized to 
detect congenital errors of metabolism $(3,4)$. The underlying concept in diagnosing congenital metabolic diseases is that the quantification of OAs and other metabolites can pinpoint dysfunction or even the absence of an enzyme due to a genetic defect (Fig. 1). Accordingly, the dysfunctioning or missing enzyme may cause metabolite accumulation in the related pathway $(6,7)$. In the early seventies, Horning and Horning, first described the term metabolic profile (8). Moreover, the research efforts of Horning's team (8) in parallel with those of Pauling et al led to the use of gas chromatography-mass spectrometry (GC-MS) for the quantification of metabolites in urine (9). This was the beginning of the use of metabolomics as a personalized approach in individuals not suffering from congenital metabolic diseases (10). Further developments in the field allowed for the quantification of OAs to become a fast-growing systems biology approach. Thus, in 2004, the human metabolome project was launched to identify all human metabolites, and its first version was released in 2007 (11). The metabolome is not only a simpler and more reflective index of health status, but it also incorporates environmental influences, for example, from exercise and diet, according to a Harvard Magazine article (7).

Urine OAs may reflect the activity of main metabolic pathways, and their quantification can be used to assess health status, nutritional status, vitamin deficiencies and response to pharmaceutical interventions, and may even be applied in toxicology and poisoning $(1,7,12)$. For instance, urine fumarate and urine malate levels have been found to be associated with mitochondrial disease with a $5 \%$ false positive rate (13). Given the fact that metabolomics reflects the phenotype and is sensitive to genetic, epigenetic and environmental factors, such as diet, age, lifestyle and xenobiotic exposure, it has been proposed as a useful tool for the identification of specific pathological conditions by assessing their influence on phenotype. The concentration of OAs in urine may vary in different populations due to different interactions among genetic and phenotypic factors (14). OAs have been widely used to screen inborn errors of metabolism, where the alterations in metabolite levels may be a hundred- or a thousand-fold increased in comparison to those of healthy subjects and this is reflected on the precision of reference values (RVs) currently used in laboratories $(14,15)$.

To date, a limited number of studies have focused on the generation of sensitive RVs for a healthy population. Thus, the aim of the present study was the evaluation of RVs in urine OAs in a well-defined healthy adult population.

\section{Subjects and methods}

Subjects. In this study, 122 Caucasian adults (51 males and 71 females) from Greece were included. The mean age of all the participants was $42.0 \pm 9.6$ years with a range of 21-81 years. Sampling procedures for the patients and healthy controls were defined according to the ethical guidelines of the Ethics Committee of the University of Crete (approval no. 1019/18/07_12_2016). The participants did not exhibit any symptoms of disease, showed normal results on clinical laboratory tests (hemogram and chemical constituents) and were not on a restricted diet. At the time of the analysis, no supplements or medication were taken. Early morning urine samples were collected and stored at $-80^{\circ} \mathrm{C}$ until analysis.
Table I. Urine OA metabolites and their validated analytical parameters.

Precision

Analyte (cv\% duplicates) Linearity Recovery

\begin{tabular}{lrlr}
\hline 2-OH Glutaric acid & 4.4 & 1.0 & 101 \\
3-Methylglutaric acid & 9.7 & 1.0 & 112 \\
3-OH-3 methylglutaric acid & 11.2 & 1.0 & 55 \\
3-OH Isobutyric & 14.8 & 0.99 & 78 \\
3-OH Isovaleric & 64.1 & 0.84 & 102 \\
4-OH Butyric & 12.0 & 1.0 & 63 \\
Adipic acid & 6.4 & 1.0 & 114 \\
Glyceric acid & 9.7 & 1.0 & 79 \\
Ethylmalonic acid & 5.1 & 1.0 & 100 \\
Fumaric acid & 9.2 & 1.0 & 87 \\
Glutaric acid & 1.2 & 1.0 & 101 \\
Glycolic acid & 13.7 & 0.99 & 86 \\
Hexanoylglycine & 16.5 & 0.98 & 109 \\
2-Ketoglutaric acid & 15.5 & 1.0 & 84 \\
Malic acid & 30.4 & 0.99 & 76 \\
Methylmalonic acid & 5.0 & 1.0 & 104 \\
Mevalonic acid & 57.6 & 0.92 & 49 \\
Pyroglutamic acid & 4.5 & 1.0 & 82 \\
Sebacic acid & 7.3 & 1.0 & 105 \\
Suberic acid & 6.5 & 1.0 & 101 \\
Tiglylglycine & 5.8 & 1.0 & 92 \\
Vanilactic acid & 10.3 & 0.99 & 88 \\
\hline SA & & &
\end{tabular}

OA, organic acid.

GC-MS, as previously described by Tanaka et al (16), was used to identify 22 different OAs (Table I). In brief, OAs were extracted from urine by liquid-liquid extraction after mixing the specimen with an internal standard solution. The oxidation of 2-keto acids with hydroxylamine hydrochloride was performed. OAs were converted to their corresponding trimethylsilyl (TMS) ethers with $N, O$,-bis-(trimethylsilyl) trifluoroacetamide (BSTFA) containing 1\% trimethylchlorosilane (TMCS) (both from Supelco, Bellefonte, PA, USA). The derivatization imparts volatility to the OAs, which is required for GC-MS analysis. The OA-TMS ethers were separated in a capillary gas chromatography column containing an immobilized, non-polar stationary phase. Following chromatographic separation, OAs were routinely detected by electron impact mass spectrometry performed in the scan mode with a mass range between $\mathrm{m} / \mathrm{z}$ 50 and 550. Identification was achieved by comparison to published spectra compounds and quantification by comparison to the calibration of pure standard compounds in ratio to an internal standard.

Statistical analysis. A list of descriptive statistics for tendency (means, median, min and max) and dispersion (standard deviation, standard error of mean, percentiles and interquartile range) were used for describing the levels of OAs. The physiological values of OAs were set using $95 \%$ confidence intervals of the 
Table II. The concentration percentiles for specific metabolites.

\begin{tabular}{|c|c|c|c|c|c|c|c|}
\hline Urine OA metabolites & Detected (>LOD) & Mean & SD & Min-Max & $\begin{array}{l}\text { Estimated } \\
95 \% \text { CI }\end{array}$ & $\begin{array}{l}\text { Known } \\
95 \% \mathrm{CI}\end{array}$ & $\begin{array}{c}\text { No of cases } \\
\text { outside } 95 \% \mathrm{CI}^{\mathrm{a}}\end{array}$ \\
\hline 5-HIAA & $92.6 \%$ & 4.9 & 9.3 & $1.0-61.8$ & $0.0-23.4$ & $0-7.0$ & $4(13)$ \\
\hline 3-OH Pr & $96.7 \%$ & 8.0 & 8.0 & $1.0-54.4$ & $0.0-23.9$ & & \\
\hline Pim & $42.6 \%$ & 1.8 & 0.8 & $1.0-4.0$ & $0.1-3.4$ & & \\
\hline EMA & $97.5 \%$ & 2.2 & 1.7 & $1.0-10.1$ & $0.0-5.5$ & $0.4-4.2$ & $9(13)$ \\
\hline 3-OH Glut & $51.6 \%$ & 1.1 & 0.3 & $1.0-2.6$ & $0.5-1.6$ & $1.0-10.0$ & $3(0)$ \\
\hline HVA & $92.6 \%$ & 2.2 & 1.4 & $1.0-8.7$ & $0.0-4.9$ & & \\
\hline Glyc & $77.0 \%$ & 2.7 & 6.2 & $1.0-44.6$ & $0.0-14.9$ & $0.0-3.0$ & $2(2)$ \\
\hline Pyrog & $100.0 \%$ & 27.8 & 15.5 & $1.7-103.4$ & $0.0-58.6$ & & \\
\hline 3-OH Isob & $99.2 \%$ & 10.3 & 8.6 & $2.0-78.0$ & $0.0-27.4$ & & \\
\hline 2-OH Glut & $96.7 \%$ & 3.4 & 2.3 & $1.0-18.0$ & $0.0-7.9$ & & \\
\hline ICit & $98.4 \%$ & 5.2 & 5.9 & $1.3-61.4$ & $0.0-16.9$ & $16.0-99.0$ & $2(0)$ \\
\hline Cit & $100.0 \%$ & 88.7 & 75.9 & $2.0-509.5$ & $0.0-239.0$ & $0.0-656.0$ & $3(0)$ \\
\hline Mal & $82.0 \%$ & 1.1 & 0.3 & $1.0-2.5$ & $0.6-1.6$ & $0.0-5.3$ & $5(0)$ \\
\hline Suc & $89.3 \%$ & 4.8 & 7.1 & $1.0-64.0$ & $0.0-19.0$ & $29.0-87.0$ & $3(0)$ \\
\hline 2-ketog & $100.0 \%$ & 18.9 & 19.2 & $1.0-145.8$ & $0.0-57.0$ & $41.0-82.0$ & $5(112)$ \\
\hline Pyr & $100.0 \%$ & 9.6 & 6.9 & $2.0-55.2$ & $0.0-23.2$ & $3.5-22.0$ & $4(12)$ \\
\hline $\mathrm{Lac}$ & $100.0 \%$ & 21.8 & 86.1 & $2.1-926.6$ & $0.0-192.2$ & $20.0-101.0$ & $2(101)$ \\
\hline Metmal & $90.2 \%$ & 1.4 & 0.8 & $1.0-6.0$ & $0.0-3.0$ & $0.0-1.0$ & $5(43)$ \\
\hline 3-MetGlut & $88.5 \%$ & 2.1 & 1.4 & $0.8-7.3$ & $0.0-4.8$ & & \\
\hline
\end{tabular}

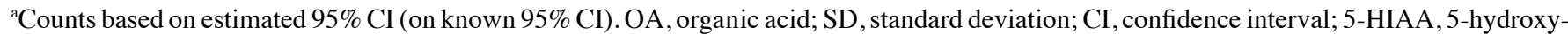
indole acetic acid; 3-OH Pr, 3-hydroxy propionic acid; Pim, pimelic acid; EMA, ethylmalonic acid; 3-OH Glut, 3-hydroxyglutaric acid; HVA, homovanillic acid; Glyc, glyceric acid; Pyrog, pyroglutamic acid; 3-OH Isob, 3-hydroxyisobutyric acid; 2-OH Glut, 2-hydroxyglutaric acid; ICit, isocitric; Cit, citric acid; Mal, malic acid; Suc, succinic acid; 2-ketog, 2 ketoglutaric acid; Pyr, pyruvic acid; Lac, lactic acid; MetMal, methylmalonic acid; 3-MetGlut, 3-methylglutaric acid.

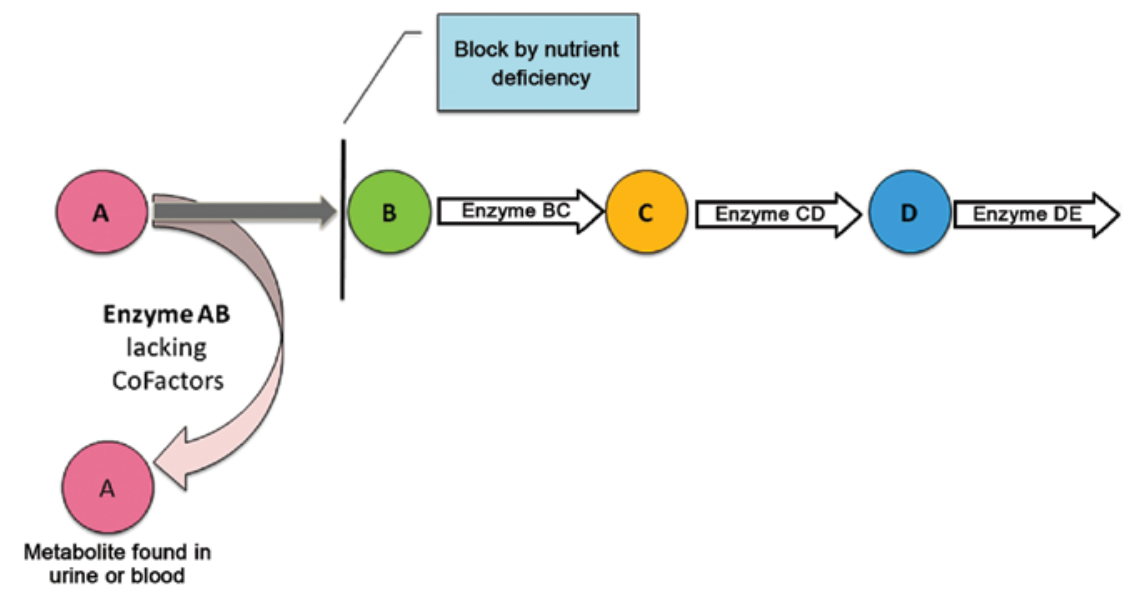

Figure 1. Micronutrient deficiency.

mean. The Kolmogorov-Smirov test was applied for examining the data normality. It has been previously demonstrated that 'normalization to partial sums' (or similar methods) has better performance as compared to normalization to creatinine concentration (17). Differences in OA levels between two or more subgroups were examined using parametric (t-test, ANOVA) and non-parametric tests (Mann-Whitney, KruskallWallis). IBM SPSS Statistics 22.0 (IBM SPSS, Armonk, NY, USA) was used for analysis of the data.

\section{Results}

The chemical analysis characteristics (precision, linearity and recovery) of 22 selected urine OAs are documented in Table I. To determine the RVs for each metabolite, the $5 \%$ percentile lower limit and $95 \%$ percentile upper limit was defined. In some cases, to achieve a more effective clinical sensitivity, changes in determining the lower and upper RVs were applied (18). The detection limit of the urine OA concentration is $1 \mu \mathrm{mol} / \mathrm{l}$. 
Table III. Percentiles and quartiles of urine OAs.

\begin{tabular}{|c|c|c|c|c|c|c|c|}
\hline & \multicolumn{3}{|c|}{ 1st quartile } & \multirow{2}{*}{$\frac{\begin{array}{c}\text { 2nd quartile } \\
\text { (median) }\end{array}}{\text { 50th percentile }}$} & \multicolumn{3}{|c|}{ 3rd quartile } \\
\hline & 5th percentile & 10th percentile & 25 th percentile & & 75 th percentile & 90th percentile & 95th percentile \\
\hline 5-HIAA & 1.0 & 1.0 & 1.5 & 2.0 & 4.3 & 8.1 & 16.6 \\
\hline 3-OH Pr & 1.6 & 1.9 & 3.3 & 5.0 & 10.2 & 18.6 & 25.5 \\
\hline Pim & 1.0 & 1.0 & 1.1 & 1.3 & 2.6 & 3.0 & 3.0 \\
\hline EMA & 1.0 & 1.0 & 1.1 & 1.5 & 2.3 & 4.8 & 6.2 \\
\hline 3-OH Glut & 1.0 & 1.0 & 1.0 & 1.0 & 1.0 & 1.2 & 1.8 \\
\hline HVA & 1.0 & 1.0 & 1.3 & 1.7 & 2.7 & 3.8 & 5.1 \\
\hline Glyc & 1.0 & 1.0 & 1.6 & 2.0 & 2.0 & 2.0 & 2.0 \\
\hline Pyrog & 5.9 & 12.0 & 18.3 & 26.0 & 33.6 & 43.1 & 55.5 \\
\hline 3-OH Isob & 2.9 & 4.6 & 5.2 & 7.8 & 13.0 & 18.0 & 22.7 \\
\hline 2-OH Glut & 1.0 & 1.3 & 1.9 & 2.8 & 4.2 & 5.6 & 7.5 \\
\hline ICit & 1.7 & 2.3 & 3.0 & 4.3 & 5.5 & 8.5 & 10.0 \\
\hline Cit & 19.8 & 26.8 & 43.8 & 65.3 & 115.6 & 171.0 & 204.7 \\
\hline Mal & 1.0 & 1.0 & 1.0 & 1.0 & 1.0 & 1.2 & 2.0 \\
\hline Suc & 1.2 & 1.4 & 1.9 & 2.8 & 5.1 & 8.9 & 11.6 \\
\hline 2-ketog & 2.4 & 2.9 & 6.5 & 13.2 & 25.8 & 39.4 & 52.2 \\
\hline Pyr & 2.9 & 4.0 & 5.4 & 8.4 & 11.5 & 16.6 & 21.1 \\
\hline Lac & 3.3 & 3.6 & 5.0 & 8.4 & 13.8 & 26.3 & 54.6 \\
\hline Metmal & 1.0 & 1.0 & 1.0 & 1.0 & 1.5 & 2.6 & 3.0 \\
\hline 3-MetGlut & 1.0 & 1.0 & 1.1 & 1.8 & 2.4 & 4.2 & 5.0 \\
\hline
\end{tabular}

OA, organic acids; 5-HIAA, 5-hydroxyindole acetic acid; 3-OH Pr, 3-hydroxypropionic acid; Pim, pimelic acid; EMA, ethylmalonic acid; 3-OH Glut, 3-hydroxyglutaric acid; HVA, homovanillic acid; Glyc, glyceric acid; Pyrog, pyroglutamic acid; 3-OH Isob, 3-hydroxyisobutyric acid; 2-OH Glut, 2-hydroxyglutaric acid; ICit, isocitric; Cit, citric acid; Mal, malic acid; Suc, succinic acid; 2-ketog, 2 ketoglutaric acid; Pyr, pyruvic acid; Lac, lactic acid; MetMal, methylmalonic acid; 3-MetGlut, 3 methyl glutaric acid.

Our study population consisted of 122 volunteers of which 71 were females $(58.2 \%)$. The mean age of all the participants was $42.0 \pm 9.6$ years with a range of $21-81$ years. There was no significant difference in age between the sexes. For analytical purposes and with respect to age, the participants were divided into 4 groups ( $\leq 30,30-40,40-50$ and $50+$ ) consisting of 18 , 28,56 and 20 subjects, respectively. Table II shows the basic descriptive parameters of the analyzed OA urine levels and the estimated physiological $95 \%$ confidence intervals. A zero concentration was set for $95 \%$ lower limit of urine OA concentration when a negative concentration was estimated. Only 3 of the urine OAs [pimelic acid (Pim), 3-hydroxyglutaric acid (3-OH Glut) and malic acid (Mal)], showed a positive (non-zero) $95 \%$ percentile lower limit. In Table III the 5th, 10th, 20th, 25th, 50th (median), 75th, as well as the 90th percentile of specific metabolites are presented. The mean levels of OAs over the age groups exhibited no significant differences $(\mathrm{P}>0.05)$ among the females and males (Table IV).

Moreover, Pearson's and Spearman's rho coefficients were applied to estimate bivariate associations between urine OAs (Table V). Of note, there was a positive correlation $(\mathrm{P}<0.05)$, between the levels of discrete OA metabolites (Table V). Thus, homovanillic acid (HVA) correlated with all of OAs except Pim, 3-OH Glut, glyceric acid (Glyc) and Mal. Similar correlations were detected for ethylmalonic acid (EMA). By contrast,
Mal only correlated significantly, but with a moderate intensity with methylmalonic acid (MetMal) (rs=0.22), 3-hydroxyisobutyric acid (3-OH Isob) (rs=0.22) and 3-hydroxypropionic acid (3-OH Pr) (rs=0.20).

\section{Discussion}

The rising significance and potential utilization of metabolomics details illustrated by the fact that since the year 2000 , 12.272 articles have been published containing the word 'Metabolomics' (Fig. 2). Importantly, it has been established that alterations in the levels of the metabolites in the quantified metabolic pathways correlate with the patient clinical status. In particular, the citric acid cycle (CDC)-metabolites in healthy individuals reflect the performance of the enzymes, which catalyze specific reactions (Fig. 3). Thus, according to studies where CDC metabolites were quantified and correlated to mitochondrial dysfunction in chronic diseases, it was shown that the presence of CDC antagonists or the lack of specific nutrients can alter CDC enzyme efficiency, and may thus provide vital information on the causative nature of a disease in a healthy North Indian pediatric population (14). Indeed, mitochondrial dysfunction has been associated with a number of chronic diseases. Heavy metals, such as mercury, can disrupt acotinase for example, in an antagonistic manner. The lack of fumarase 


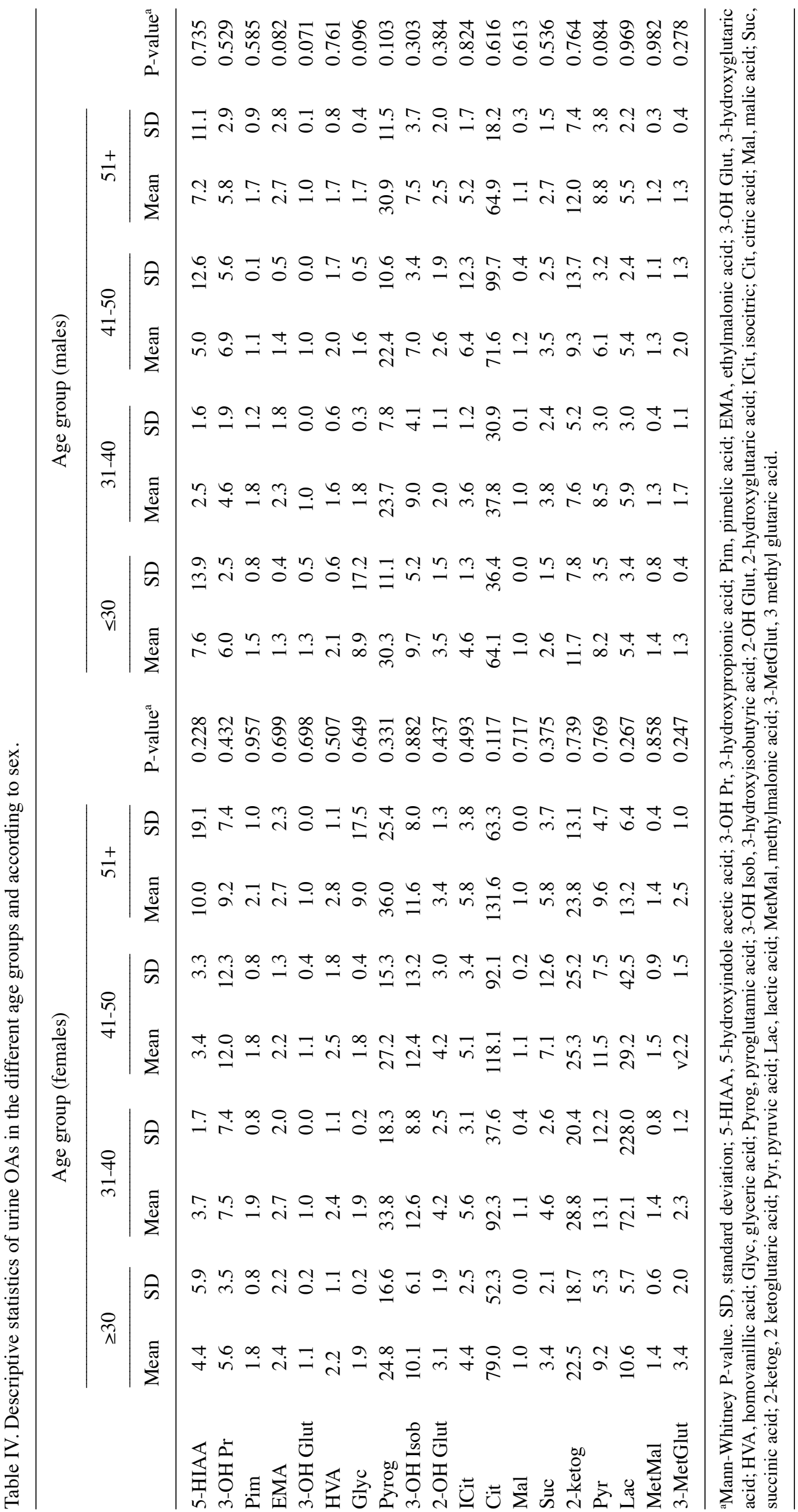


$\stackrel{0}{0}$

$\frac{4}{1}$

ש

क

$\bar{\Sigma}$

$: *$

$\ddot{0}$

节

궁

응

需

00

:

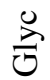

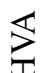

春

$\stackrel{1}{0}$

m

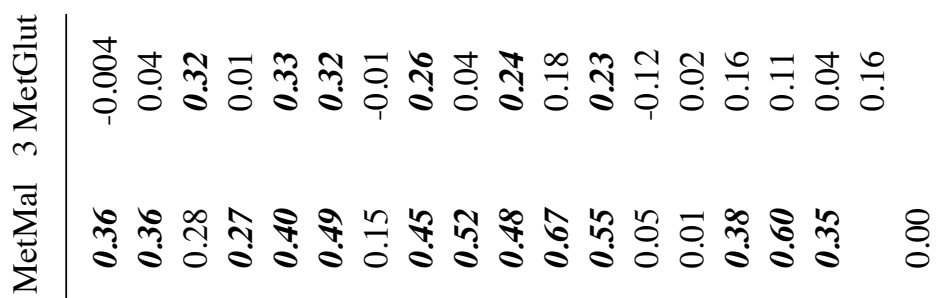

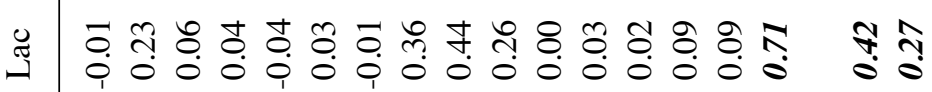

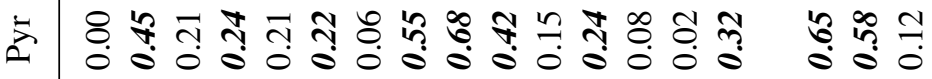

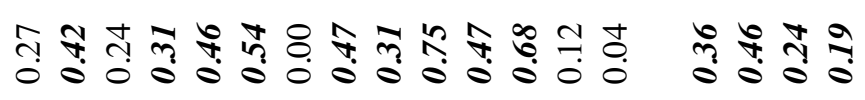

Tृ:

范

宁药

$\because \ddot{0}$

过

$\frac{0}{\circ} \cdot \frac{G}{1}$

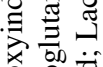

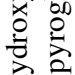

完 80.0

庭究

尾获

它. 过

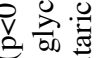

过

绻

递范

范

苞魚产

晋 $\overrightarrow{0}$

8 .

离这志

तิ

苞苛

宗

年

믈.

.

究

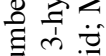

艺

$\dot{0} \cdot \stackrel{0}{g}$

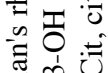

范

$m \pi n-\infty \pi \infty n$

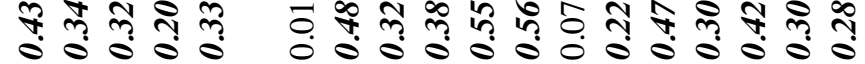

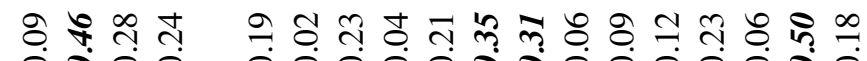

के

is .

䜦

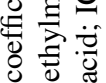

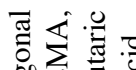

$\sum_{\infty} \sum_{I 1} \frac{\pi}{\tilde{J}}$

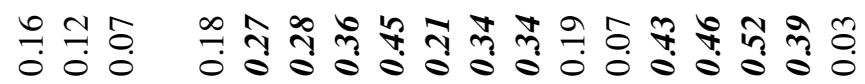

m.

$\because \ddot{x}$

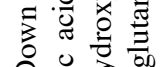

ค.ำ

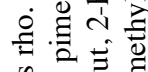

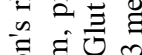

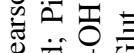

ㄴ.

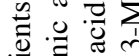

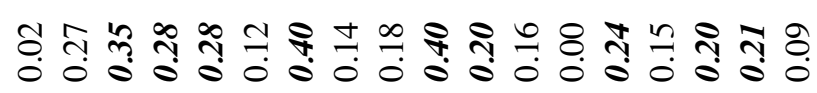

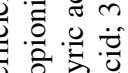

过

要通.

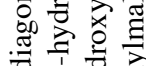

के ले 


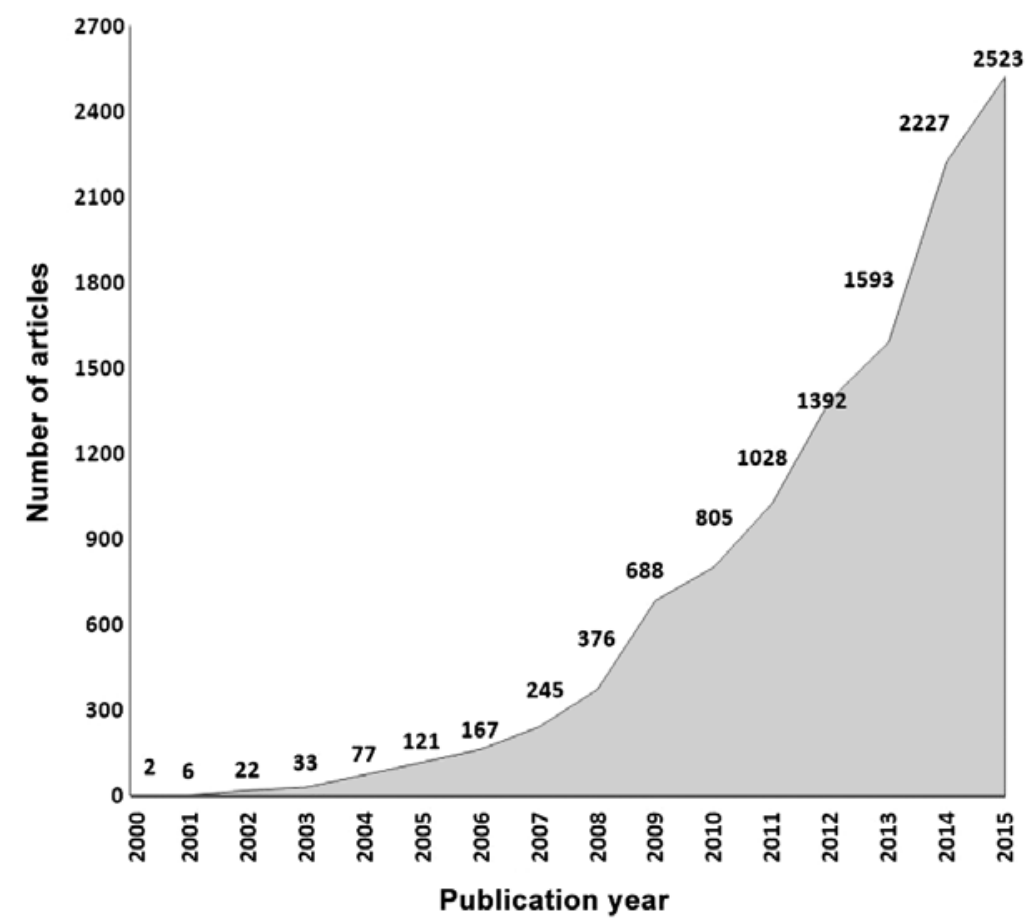

Figure 2. PubMed metabolomics articles published in the period 2000-2015.

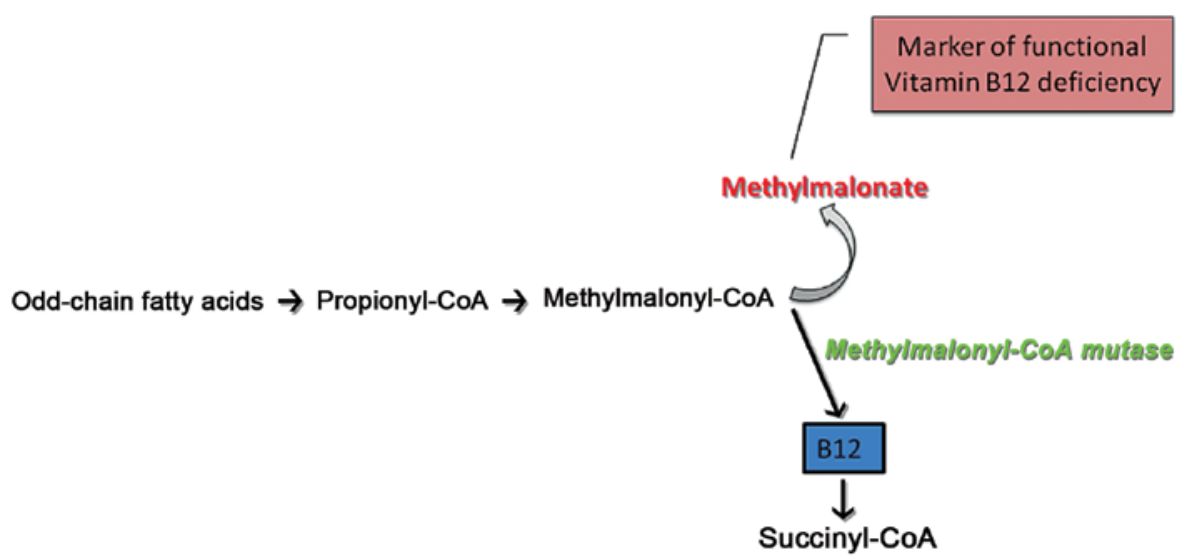

Figure 3. Methylation Marker-Vitamin B12 deficiency.

co-factors, such as ubiquinol may disrupt fumarate conversion to malate with concurrent fumarate elevation (19). Another important application of the measurement of OAs is the case of 3-hydroxybutyric acid, the levels of which increase in urine due to the failure of glucose utilization in both fasting and diabetic subjects, and can thus be used as a marker for subclinical ketoacidosis, insulin sensitivity and complications associated with diabetes $(13,20)$. In a recent clinical study, children presenting with non-alcoholic fatty liver disease (NAFLD) were treated with probiotic VSL\#3 and subsequently their urinary OAs, including amino-acid metabolites and nucleic acid degradation metabolites were estimated. The data obtained in that study suggested that the urinary OAs levels of children with NAFLD may be utilized as efficient biomarkers to evaluate the response to therapy (21). It has recently been discussed that both genetic and biochemical data indicate a contribution of the mitochondrial disease during the pathological evolvement of autism spectrum disorder (ASD) in a specific subgroup of children (22). Indeed, significant differences in urinary 4-hydroxyphenylacetic, 2-oxoglutaric, isocitric, citric, 4-hydroxybenzoic, hippuric, adipic and suberic levels have been detected in autistic children, as compared to age-matched healthy controls $(23,24)$. Furthermore, urine homovanillic acid and vanilmandelic acid levels have shown to be related to neurotransmitter metabolism in subjects suffering from occupational stress (25). Moreover, a utilization of metabolic profiling for the detection of early chronic kidney disease has recently been highlighted. Such an approach could significantly decrease the existing time lapse between the onset of the pathology and the time point when symptoms are of adequate intensity as to initiate therapy (25). Moreover, metabolic profiling, including the evaluation of 2-hydroxyisobutyric acid, has been suggested as a feasible strategy in the evaluation of renal disease progression (26). A separate study demonstrated a correlation between urinary 
amino acid metabolites' levels and chronic kidney disease progression (27). Moreover, changes in arginine metabolism have likewise been indicated as a biomarker for the 2, 3 and 4 stages of chronic kidney disease (28). As these patients constitute a significant subgroup of total human pathologies, improved early detection and accurate disease monitoring could increase the efficiency of treatment and significantly improve the socioeconomic aspects of disease. Another feasible application of metabolic profiling is the evaluation of intestinal disease. Thus, 4-hydroxyphenylacetic acid, an intestinal bacterial flora metabolite, was found to be a marker of small-bowel disease due to bacterial overgrowth as and in the case of Giardia infestation (29). The utilization of OAs as disease markers is still limited due to the lack of stringent, reproducible RVs of healthy controls, as well as to difficulties when comparing data from different platforms (30). At present, RVs are mainly based on studies and measurements performed with the aim to detect inborn errors of metabolism (31). In patients presenting with congenital metabolic diseases, the lack or substantial dysfunction of an enzyme participating in a specific metabolic pathways markedly alters the levels of the metabolites involved, allowing the use of a wider interval for RVs. However, in patients suffering from a metabolic pathway dysfunction due to epigenetic factors, there are smaller variations of the metabolite levels between the healthy controls and subjects with pathologies. This, demands strictly defined RVs in order to detect and correctly interpret minor to moderate deviations. The human metabolome database has contributed significantly to the increased application of metabolic profiling in clinical settings. Moreover, the accumulation of high resolution metabolomics data from a variety of platforms is approaching the critical threshold in assembling a central, reference human metabolome $(11,30)$. This study, therefore aimed to qualitatively contribute to existing data bases of urine metabolites with the utmost goal being to increase sensitivity in detecting metabolic pathway deviations in chronic diseases.

Over the past 6 years, we have performed more than 15,000 OA profiles, targeting metabolites that relate to critical metabolic pathways, such as the CDC, neurotransmitter turnover, bacterial flora markers, protein metabolism, ketones metabolism, fatty acid $\beta$-oxidation methylation markers, vitamin B sufficiency and carbohydrate metabolism. Therefore, according to our experience, more sensitive RVs are required to identify smaller variations in individuals presenting with various pathologies but not suffering from inborn errors of metabolism.

This study was designed to determine the RV for a set of key OA metabolites in a population of healthy Greek adult males and females, who were on an unrestricted diet. The establishment of a successful analytical profiling technique involves the simultaneous analysis of numerous metabolites with various chemical characteristics. Furthermore, the separation, identification and quantification of these metabolites need to be performed with the highest possible accuracy. It is imperative to be able to detect very low concentrations of metabolites in order to detect any deviation with respect to normal levels. According to the above-mentioned characteristics, the technical method of choice needs to exhibit high sensitivity and repeatability. In previous studies, the analytical techniques that have been used are GC-MS, HPLC, TANDEM-MS and others. However, the gold standard for the evaluation of OAs in urine is GC-MS with the chemical modification of metabolites, separated by chromatography and identified by mass spectrometry (32-34). Prior to application in each respective laboratory, the method needs to be validated with specific conclusions about the value ranges, the repeatability and the linearity of measurements. This methodology needs to be carefully applied for the obtaining of accurate and representative $\mathrm{RV}$. To the best of our knowledge, this study is the first of its kind conducted in Greece. The concentrations of OAs may vary among populations from different geographical regions due to genotype, different diet and various other environmental and epigenetic factors. Moreover, there is extensive individual variation in metabolite secretion following specific dietary intake (28). Along these lines, our results are in agreement with those of similar studies performed on different ethnic groups with minor differences attributed to the details of laboratory methods (35). A recent study performed on a healthy north Indian pediatric population utilizing mass GS-MS indicated that the concentrations of OAs were higher (14) as compared to matched age-groups in Turkish and Swiss $(36,37)$ pediatric populations. Thus, Kumari et al, putatively attribute the higher RVs of some metabolites to dietary deficiency, as protein intake was inadequate in the North Indian pediatric population (14). Moreover, studies on pediatric populations have shown a strong age dependent effects on RVs of OA metabolites $(14,37)$.

Our study contributes to the available data with the aim to narrow the RV range, and the increase sensitivity and the clinical application of urine metabolites in detecting deviation from normal metabolism. However, further studies with well-defined groups of patients presenting specific symptoms or diseases, are warranted in order to test the ability of this method to discriminate between normal and pathological values.

\section{References}

1. Astarita $\mathrm{G}$ and Langridge $\mathrm{J}$ : An emerging role for metabolomics in nutrition science. J Nutrigenet Nutrigenomics 6: 181-200, 2013.

2. Baraldi E, Carraro S, Giordano G, Reniero F, Perilongo G and Zacchello F: Metabolomics: Moving towards personalized medicine. Ital J Pediatr 35: 30, 2009.

3. Sun J, Beger DR and Schnackenberg KL: Metabolomics as a tool for personalizing medicine: 2012 update. Per Med 10: 149-161, 2013.

4. McKillop AM and Flatt PR: Emerging applications of metabolomic and genomic profiling in diabetic clinical medicine. Diabetes Care 34: 2624-2630, 2011.

5. Tanaka Kand Isselbacher KJ:Experimental beta-hydroxyisovaleric aciduria induced by biotin deficiency. Lancet 2: 930-931, 1970.

6. van der Greef $\mathbf{J}$ and Smilde AK: Symbiosis of chemometrics and metabolomics: past, present, and future. Chemometrics 19: 376-386, 2005

7. Shaw J: Fathoming Metabolism. The study of metabolites does an end run around genomics to provide telling clues to your future health. Harv Mag 3: 27-31, 2011.

8. Horning EC and Horning MG: Metabolic profiles: gas-phase methods for analysis of metabolites. Clin Chem 17: 802-809, 1971.

9. Pauling L, Robinson AB, Teranishi R and Cary P: Quantitative analysis of urine vapor and breath by gas-liquid partition chromatography. Proc Natl Acad Sci USA 68: 2374-2376, 1971.

10. Griffiths WJ and Wang Y: Mass spectrometry: From proteomics to metabolomics and lipidomics. Chem Soc Rev 38: 1882-1896, 2009.

11. Wishart DS, Tzur D, Knox C, Eisner R, Guo AC, Young N, Cheng D, Jewell K, Arndt D, Sawhney S, et al: HMDB: The Human Metabolome Database. Nucleic Acids Res 35 (Database): D521-D526, 2007. 
12. Jones PM and Bennett MJ: Urine organic acid analysis for inherited metabolic disease using gas chromatography-mass spectrometry. Methods Mol Biol 603: 423-431, 2010.

13. Barshop BA: Metabolomic approaches to mitochondrial disease: Correlation of urine organic acids. Mitochondrion 4: 521-527, 2004

14. Kumari C, Singh A, Ramji S, Shoemaker JD and Kapoor S: Urinary Organic Acids Quantitated in a Healthy North Indian Pediatric Population. Indian J Clin Biochem 30: 221-229, 2015.

15. Fiehn O and Spranger J: Use of metabolomics to discover metabolic patterns associated with human disease. Pulm Circ 3 : 417-423, 2014

16. Tanaka K, Hine DG, West-Dull A and Lynn TB: Gas-chromatographic method of analysis for urinary organic acids. I. Retention indices of 155 metabolically important compounds. Clin Chem 26: 1839-1846, 1980.

17. Applegarth DA and Ross PM: The unsuitability of creatinine excretion as a basis for assessing the excretion of other metabolites by infants and children. Clin Chim Acta 64: 83-85, 1975

18. Blau N, Duran M and Gibson KM: Laboratory Guide to the Methods in Biochemical Genetics. Springer, Berlin Heidelberg, pp7-22, 2008.

19. Mitchell P: Possible molecular mechanisms of the protonmotive function of cytochrome systems. J Theor Biol 62: 327-367, 1976.

20. Yousri NA, Mook-Kanamori DO, Selim MM, Takiddin AH, Al-Homsi H, Al-Mahmoud KA, Karoly ED, Krumsiek J, Do KT, Neumaier U, et al: A systems view of type 2 diabetes-associated metabolic perturbations in saliva, blood and urine at different timescales of glycaemic control. Diabetologia 58: 1855-1867, 2015.

21. Miccheli A, Capuani G, Marini F, Tomassini A, Praticò G, Ceccarelli S, Gnani D, Baviera G, Alisi A, Putignani L, et al: Urinary (1)H-NMR-based metabolic profiling of children with NAFLD undergoing VSL \#3 treatment. Int J Obes 39: 1118-1125, 2015.

22. Legido A, Jethva $\mathrm{R}$ and Goldenthal MJ: Mitochondrial dysfunction in autism. Semin Pediatr Neurol 20: 163-175, 2013.

23. Noto A, Fanos V, Barberini L, Grapov D, Fattuoni C, Zaffanello M, Casanova A, Fenu G, De Giacomo A, De Angelis M, et al: The urinary metabolomics profile of an Italian autistic children population and their unaffected siblings. J Matern Fetal Neonatal Med 27 (Suppl 2): 46-52, 2014.

24. Kałużna-Czaplińska J: Noninvasive urinary organic acids test to assess biochemical and nutritional individuality in autistic children. Clin Biochem 44: 686-691, 2011.

25. Wu H, Jiang $\mathrm{K}, \mathrm{Gu} \mathrm{G}, \mathrm{Wu} \mathrm{Y}$ and $\mathrm{Yu} \mathrm{S}$ : The relationship of occupational stress and the level of some hormone metabolites in urine. Zhonghua Lao Dong Wei Sheng Zhi Ye Bing Za Zhi 32: 83-86, 2014 (In Chinese)
26. Mutsaers HA, Engelke UF, Wilmer MJ, Wetzels JF, Wevers RA, van den Heuvel LP, Hoenderop JG and Masereeuw R: Optimized metabolomic approach to identify uremic solutes in plasma of stage 3-4 chronic kidney disease patients. PLoS One 8: e71199, 2013.

27. Duranton F, Lundin U, Gayrard N, Mischak H, Aparicio M, Mourad G, Daurès JP, Weinberger KM and Argilés A: Plasma and urinary amino acid metabolomic profiling in patients with different levels of kidney function. Clin J Am Soc Nephrol 9: 37-45, 2014.

28. Shah VO, Townsend RR, Feldman HI, Pappan KL, Kensicki E and Vander Jagt DL: Plasma metabolomic profiles in different stages of CKD. Clin J Am Soc Nephrol 8: 363-370, 2013.

29. Chalmers RA, Valman HB and Liberman MM: Measurement of 4-hydroxyphenylacetic aciduria as a screening test for small-bowel disease. Clin Chem 25: 1791-1794, 1979.

30. Li S, Todor A and Luo R: Blood transcriptomics and metabolomics for personalized medicine. Comput Struct Biotechnol J 14: $1-7,2015$.

31. Nagrath D, Caneba C, Karedath T and Bellance N: Metabolomics for mitochondrial and cancer studies. Biochim Biophys Acta 1807: 650-663, 2011.

32. Duez P, Kumps A and Mardens Y: GC-MS profiling of urinary organic acids evaluated as a quantitative method. Clin Chem 42: $1609-1615,1996$

33. Greter $\mathbf{J}$ and Jacobson CE: Urinary organic acids: Isolation and quantification for routine metabolic screening. Clin Chem 33: 473-480, 1987.

34. Hoffmann G, Aramaki S, Blum-Hoffmann E, Nyhan WL and Sweetman L: Quantitative analysis for organic acids in biological samples: Batch isolation followed by gas chromatographic-mass spectrometric analysis. Clin Chem 35: 587-595, 1989.

35. Bouatra S, Aziat F, Mandal R, Guo AC, Wilson MR, Knox C, Bjorndahl TC, Krishnamurthy R, Saleem F, Liu P, et al: The human urine metabolome. PLoS One 8: e73076, 2013.

36. Kiykim E, Zeybek CA, Zubarioglu T, Cansever S, Yalcinkaya C, Soyucen E and Aydin A: Inherited metabolic disorders in Turkish patients with autism spectrum disorders. Autism Res 9: 217-223, 2016.

37. Boulat O, Gradwohl M, Matos V, Guignard JP and Bachmann C: Organic acids in the second morning urine in a healthy Swiss paediatric population. Clin Chem Lab Med 41: 1642-1658, 2003. 